\title{
Synthesis and antimicrobial activity of novel 2,4-disubstituted thiazoles
}

\author{
Birutė Grybaite $^{1}$, Rita Vaickelionienè ${ }^{1}$, Vytautas Mickevičius ${ }^{1}$, \\ Viktor Zvarych ${ }^{2}$, Volodymyr Novikov ${ }^{2}$ \\ 1. Department of Organic Chemistry, Kaunas University of Technology, LITHUANIA, Kaunas, \\ Radvilènų pl. 19, E-mail: birute.grybaite@ktu.edu \\ 2. Department of Technology of Biologically Active Substances, Pharmacy and Biotechnology, \\ Lviv Polytechnic National University, UKRAINE, Lviv-13, S. Bandera Str. 12, \\ E-mail: maryna.v.stasevych@gmail.com

\begin{abstract}
A beneficial modifications of 2-substituted thiazoles by incorporating different functional groups into the structure such as carboxyl, chloromethyl, (phenylamino)methyl, ethoxyoxoethyl, which determine a certain chemical reactions of the compound was developed. All the synthesized compounds were tested for their in vitro antimicrobial and antifungal activity.
\end{abstract}

Keywords - amino acids, antimicrobial agents, cyclization, functionalized thiazoles, reactive intermediates.

\section{Introduction}

A large number of chemotherapeutics are developed for medicinal use in nowadays but the increasing resistance of pathogens to available pharmaceuticals has created an essential demand for new efficient classes of antimicrobial agents. A unique small-ring heterocycle - thiazole containing nitrogen and sulphur atoms, play an important role in medicinal chemistry and is widely used in the development of bioactive compounds, drugs, as well as industrial products [1, 2]. Thiazole derivatives are associated with a broad spectrum of biological properties, including antimicrobial [3], antituberculous [4] and anticancer [5]. The aim of this work was to synthesize functionalized thiazoles with reactive functional groups that could be intermediates for the synthesis of variously functionalized thiazoles.

\section{Results and discussion}

\section{Chemistry}

Our initial aim of this work was to synthesize a series of disubstituted aminothiazoles (Scheme 1). To give the derivatives $\mathbf{2 a}, \mathbf{b}$ the reaction of the corresponding thioureido acids $\mathbf{1 a}$, b with 1,3-dichloroacetone was carried out under reflux in acetone for $2 \mathrm{~h}$. Under alkaline hydrolysis conditions, the chloromethyl group of compounds $\mathbf{2 a}$, b was replaced by a hydroxymethyl fragment $\mathbf{3 a}, \mathbf{b}$.

Scheme 1. Synthesis of disubstituted thiazoles 2-6

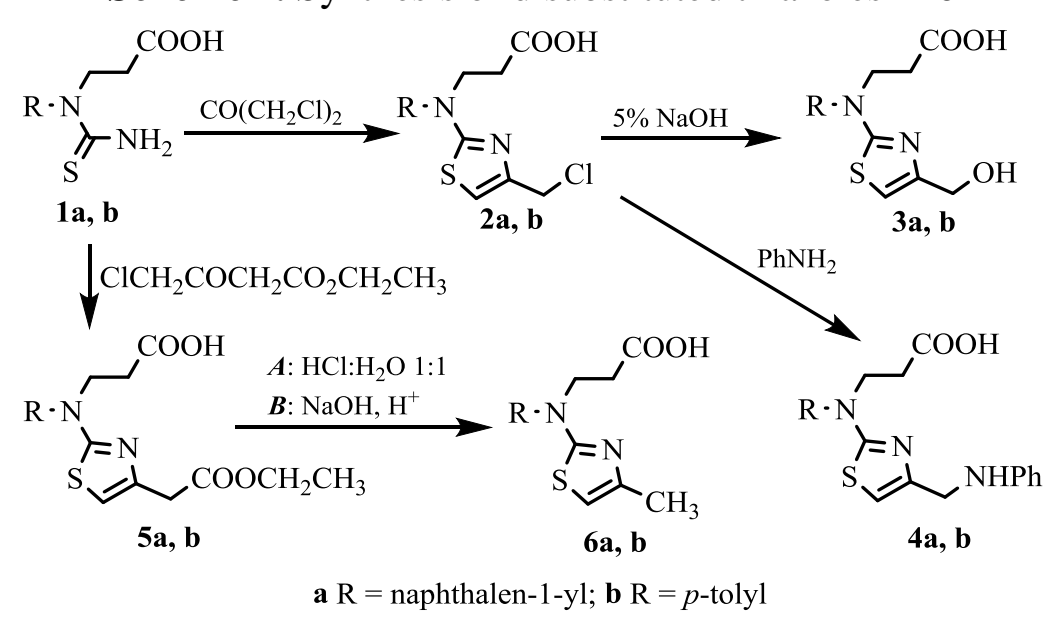


The interaction of aminothiazoles $\mathbf{2 a}, \mathbf{b}$ with aniline in dimethylformamide results in the formation of the corresponding 3-(naphthalen-1-yl(4-((phenylamino)methyl)thiazol-2yl)amino)propanoic acid (4a) and 3-((4-((phenylamino)methyl)thiazol-2-yl)(ptolyl)amino)propanoic acid (4b). Esters 5a, $\mathbf{b}$ were prepared by the reaction of corresponding thioureido acid 1a, $\mathbf{b}$ and ethyl 4-chloroacetoacetate in acetone. The attempts of the acidic or alkaline hydrolysis of the ethoxy oxoethyl moiety in $\mathbf{5 a}, \mathbf{b}$ did not succeed and led to the unexpected products 4-methylthiazole derivatives $\mathbf{6 a}, \mathbf{b}$.

\section{Biology}

All synthesized compounds 2-5 were tested against bacteria strain Staphylococcus aureus 209-P and fungi strains Candida tenuis VCM Y-70, and Aspergillus niger VCM F-1119 for their in vitro antimicrobial activity by the diffusion method in agar and the serial dilution method. Analysis of the structure-activity relationship of the synthesized compounds has revealed that derivatives with $p$-tolyl moiety are more active than naphthalenyl analogues. Majority of the most active compounds against $C$. tenuis identified by the serial dilution method are the ones bearing $p$-tolyl fragment. Compounds $\mathbf{3 b} \mathbf{b} \mathbf{5 b}$ showed equal effectiveness as Nystatin. In the case of $A$. niger, the most active appeared to be compounds containing $p$-tolyl moiety, i.e. derivatives $\mathbf{2 b}, \mathbf{4 b}$ and $\mathbf{5 b}$. The advantage of compounds with the $p$-tolyl moiety has been also demonstrated by antibacterial activity of $\mathbf{3 b}$ against S.aureus, the MIC of which corresponds to the control Vancomycin.

\section{Conclusion}

In summary, using different types of chemical reactions we have synthesized a series of thiazoles with reactive functional groups in the molecule, which allow predicting, design and formation of new thiazole structures.

\section{References}

[1] M. V. J. Nora de Souza, "Synthesis and biological activity of natural thiazoles: An important class of heterocyclic compounds", Sulfur Chem., vol. 26, no. 4-5, Aug.-Oct., pp. 429-449, 2005.

[2] P. K. Sasmal, S. Sridhar, J. Idbal, "Facile synthesis of thiazoles via an intramolecular thia-Michael strategy", Tetrahedron Lett., vol. 47, iss. 49, Dec., pp. 8661-8665, 2006.

[3] B. Grybaitè, R. Vaickelionienè, M. Stasevych, o. Komarovska-Porokhnyavets, V. Novikov, V. Mickevičius, „Synthesis, Transformation of 3-[(4-Arylthiazol-2-yl)(ptolyl)amino]propanoic Acids, Bis(thiazol-5-yl)phenyl-, Bis(thiazol-5-yl)methane Derivatives, and Their Antimicrobial Activity“, Heterocycles, vol. 96, no. 1, Dec., pp. 86-105, 2018.

[4] Y. K. Abhale, A. Shinde, K. K. Deshmukh, L. Nawale, D. Sarkar, P. C. Mhask, "Synthesis, antitubercular and antimicrobial potential of some new thiazole substituted thiosemicarbazide derivatives", Med. Chem. Res., vol. 26, iss. 10, Oct., pp. 2557-2567, 2017.

[5] T. I. de Santana, M. O. Barbosa, P. A. T. M. Gomes, A. C. N da Cruz , T. G. da Silva, A. C. L. Leite, "Synthesis, anticancer activity and mechanism of action of new thiazole derivatives", Eur. J. Med. Chem., vol. 144, Jan., pp. 874-886, 2018. 